\title{
Vehicular Ad-hoc Networks using slotted Aloha: Point-to-Point, Emergency and Broadcast Communications
}

\author{
Bartłomiej Błaszczyszyn \\ INRIA/ENS
}

\author{
Paul Muhlethaler \\ INRIA Rocquencourt
}

\author{
Nadjib Achir \\ INRIA Rocquencourt and Paris XIII
}

\begin{abstract}
The aim of this paper is to analyze the Aloha medium access (MAC) scheme in one-dimensional, linear networks, which might be an appropriate assumption for Vehicular Ad-hoc NETworks (VANETs). The locations of the vehicles are assumed to follow a homegeneous Poisson point process. Assuming powerlaw mean path-loss and independent point-to-point fading we study performance metrics based on the signal-over-interference and noise ratio (SINR). In contrast to previous studies where the receivers are at a fixed distance from the transmitter, we assume here that the receivers are the nearest neighbors of the transmitters in the Poisson process and in a given direction. We derive closed formulas for the capture probability and for the density of progress of a packet sent by a given node. We compute the mean delay to send a packet transmitted at each slot until successful reception. We also evaluate an upper bound to discover the neighborhood within a given space interval. We show that we can include noise in these models.

Index Terms-VANETs, slotted Alohaa, MAC (Medium Access
\end{abstract} Control) Layer Optimization, performance evaluation

\section{INTRODUCTION}

VANETs may well be the most promising application of Mobile Ad hoc NETworks (MANETs). Promotors of VANETs believe that these networks will both increase safety on the road and provide value-added services. Numerous challenging problems must however be solved in oder to be able to propose these services e.g. efficient and robust physical layers, reliable and flexible medium access protocols, routing schemes and optimized applications.

Although the medium access protocols envisioned for VANETs are mostly based on Carrier Sense Multiple Access (CSMA) schemes e.g; IEEE 802.11p, techniques using carrier sensing lead to complex patterns of simultaneously transmitting nodes. Thus it is difficult to build analytical models for such schemes. Moreover carrier sensing introduces many parameters that must be optimized, such as the interframes, the defer threshold, the back-off window, etc. In contrast, slotted Aloha has only one parameter: the transmission probability $p$ (Medium Access Probability MAP) and the pattern of simultaneously transmitting nodes is very simple. Thus we chose to use Aloha as a medium access scheme. Aloha has received a lot of attention in the literature and most of the studies are for two-dimensional networks. Moreover these studies usually use the bipolar model in which each node which can transmit has a receiver randomly positioned at a fixed distance from the transmitter. In contrast to this assumption, we are able to study a Signal-over-Interference and Noise Ratio (SINR) model where both the transmitter and the receiver are in the intial Poisson point process which models the locations of the vehicles.

\section{A. Main contributions}

The main contributions of this paper are the following

- For the two receiving models in this paper i.e. the nearestneighbor and the nearest-receiving neighbor in a given direction, we evaluate the probability of capture of a packet sent by a fixed node.

- With the same assumption, we compute the density of progress of such a packet and we study how to optimize this density.

- We then study the transmission delay for a safety packet which is sent by a given node and at each slot.

- We also derive an upper-bound of the delay to send a broadcast packet in a given space interval.

- We show that the above analyses can take noise into account.

\section{B. Related Work}

There is abundant literature on the performance evaluation of general MANETs under CSMA and Aloha. However, very few papers, such as [8], focus specifically on VANETs. To the best of our knowledge, most of these studies use simulations to evaluate the performances of these networks and/or assume simplified interference models. Aloha can be analyzed more easily than CSMA, even in quite a realistic SINR scenario [5, $6,9]$. However these studies assume planar (two-dimensional) networks and a Poisson point process to model the location of the network nodes. This approach was partially adapted to linear VANETs in a study [7] where it is assumed that the nodes use slotted Aloha. Numerous studies [1, 10,11] evaluate beaconing algorithms in VANETs. Another study [2] considers disconnected VANETs and evaluates DTN schemes to propagate the packets within the network. In contrast to previous papers [5-7, 9], we compute the network performances for receivers within the Poisson point process which models the location of the network nodes, whereas the previous studies assume that each transmitter has its own receiver at given distance from it.

\section{Organization of the paper}

The remaining part of this paper is organized as follows: The network and propagation models are described respectively in Sections II-A, II-B. In Section II-C the SINR capture (nonoutage) condition is described. In Section II-D the nearest neighbor models used throughout this paper are proposed. With these models we compute the probability of capture in Section II-E and the density of progress in Section II-F. In 
section II-G we compute the nearest neighbor warning delay time and in Secion II-H we compute the neighborhood discovery time. We extend the results obtained to the case where we have constant noise. Section IV discusses the numerical examples, followed by the conclusion in Section V.

\section{LINEAR VANET MODEL WITH SLOTTED ALOHA AND NEAREST-NEIGHBOR TRANSMISSIONS}

\section{A. Network model}

We assume a linear network topology, where (at a given time) nodes (vehicles) are randomly located on a straight line. The nodes are perfectly synchronized on time slots of the same duration and they try to access the channel according to the slotted Aloha scheme. For every time slot, each node, independently tosses a coin with some bias $p$ for heads, which will be referred to as the Aloha medium access probability (Aloha MAP). Nodes whose outcome is heads transmit their packets in the time slot, and the others do not transmit. The locations of the nodes are assumed not to change during the transmission since the duration of a transmission is so short. The above situation will be modeled by the marked Poisson point $\Phi=\left\{\left(X_{i}, e_{i}\right)\right\}$ with intensity $\lambda$ on the line $\mathbb{R}$, where

- $\left\{X_{i}\right\}$ denotes the (instantaneous) locations of vehicles; $\lambda$ is the mean density of nodes per unit of (route) distance,

- $\left\{e_{i}\right\}_{i}$ is the medium access indicator of node $i ; e_{i}=1$ for the node which is allowed to emit and $e_{i}=0$ for the node which is not allowed to emit. The random variables $e_{i}$ are independent, with $\mathbf{P}\left(e_{i}=1\right)=p$. Sometimes we will use the notation $e_{X_{i}}=e_{i}$.

Let us split $\Phi$ into two point processes: $\Phi^{1}=\left\{X_{i}: e_{i}=1\right\}$ representing transmitters, and $\Phi^{0}=\left\{X_{i}: e_{i}=0\right\}$ representing silent nodes. The latter are potential receivers. A basic fact from the theory of Point processes is that $\Phi^{1}$ and $\Phi^{0}$ are independent Poisson point processes of intensity $\lambda p$ and $\lambda(1-p)$ respectively.

\section{B. Signal propagation}

Each transmitting vehicle uses the same transmission power $S$. The signal-power path-loss is modeled by the power-law function $l(r)=(A r)^{\beta}$ where $r$ is the distance between the transmitter and the receiver; we will specify the receiver in section II-D. Our mathematical linear model of the network requires $\beta>1$ in order for the sum of all powers received at a given location to have a finite mean.

Signal-power is also perturbed by random fading $F$ that is independently sampled for each transmitter-receiver pair. Thus, the actual signal-power received at $y$ from $x$ is equal to $S F_{(x, y)} / l(|x-y|)$. If not specified, $F$ is assumed to have a general distribution with finite moment $\mathbf{E}\left[F^{1 / \beta}\right]$. In this section we will restrict ourselves to an important special case when $F$ is exponentially distributed, which corresponds to the situation of independent Rayleigh fading. By eventual renormalization of $S$ we can assume without loss of generality that $F$ has a mean of 1 . Moreover in this section we do not consider external noise ( $W=0$ ) but we keep $W$ in the notation for the remaining part of this paper.

\section{Reception}

When a vehicle located at $x$ transmits a signal with power $S$ and this signal is being received by a vehicle located at $y$, then the success of this reception depends on the signal-tointerference-and-noise ratio (SINR)

$$
\operatorname{SINR}_{(x, y)}=\frac{S F_{(x, y)} / l(|x-y|)}{W+I_{\Phi^{1}}(y)},
$$

where $I_{\Phi^{1}}$ is the shot-noise process of $\Phi^{1}: I_{\Phi^{1}}(y)=$ $\sum_{X_{i} \in \Phi^{1}} S F_{\left(y, X_{i}\right)} / l\left(\left|y-X_{i}\right|\right)$. In this paper we assume a fixed bit-rate coding, i.e., $y$ successfully receives the signal from $x$ if

$$
\operatorname{SINR}_{(x, y)} \geq T,
$$

where $\operatorname{SINR}_{(x, y)}$ is given by (2.1) and $T$ is the SINR-threshold related to the bit-rate given some particular coding scheme.

\section{Nearest neighbor receiver models}

A particular choice of receivers depends on the routing scheme implemented in the network. Motivated by the usual routing mechanisms which typically select relay nodes in the vicinity of the transmitters, in this section we consider the two following receiver models:

- Nearest Neighbor in a given Direction (NND). We assume that each transmitter in $\Phi^{1}$ (cf. the network model in Section II-A) picks the nearest node to itself in $\Phi$ in the randomly selected direction (to the right or to the left on the line) as its receiver, regardless of whether or not the latter is authorized to transmit at the same time. Successful reception requires that this selected node is not authorized by Aloha to transmit (i.e, is in $\Phi^{0}$ ), and that the SINR condition (2.2) for this transmitter-receiver pair is satisfied.

- Nearest Receiver in a given Direction (NRD). Each transmitter in $\Phi^{1}$ picks the nearest node in $\Phi^{0}$ in a randomly selected direction, i.e., it picks a node that is not authorized by Aloha to transmit.

Remark that the NND model corresponds to the usual separation of routing and MAC layers, while the NRD model might be seen as corresponding to an opportunistic routing. As we will see, in linear networks both models allow for quite explicit performance analysis and, in particular, an optimal tuning of the Aloha parameter $p$, provided the external noise can be ignored $(W=0)$.

\section{E. Capture probability}

For arbitrary $a, b>0$ denote $C(a, b)=\int_{a}^{\infty} 1 /\left(u^{b}+1\right) \mathrm{d} u$ and

$$
C(b)=C(0, b)=\frac{\pi}{b \sin (\pi / b)} .
$$

Moreover, for given $T$ and $\beta$ let us denote:

$$
\begin{aligned}
& \mathcal{C}_{1}=\mathcal{C}_{1}(T, \beta)=T^{1 / \beta}\left(C\left(T^{-1 / \beta}, \beta\right)+C(\beta),\right. \\
& \mathcal{C}_{2}=\mathcal{C}_{2}(T, \beta)=2 T^{1 / \beta} C(\beta)=\frac{2 T^{1 / \beta} \pi}{\beta \sin (\pi / \beta)} .
\end{aligned}
$$

Proposition 2.1: The probability of successful transmission for slotted Aloha with MAP $p$ in the NND receiver model with 
exponential fading and negligible noise $(W \equiv 0)$ is equal to:

$$
P_{N N D}(p)=\frac{1-p}{1+p \mathcal{C}_{1}}
$$

and in the NRD receiver model

$$
P_{N R D}(p)=\frac{1-p}{1+p\left(\mathcal{C}_{2}-1\right)} .
$$

Proof: Note directly from the form of the SINR in (2.1) that $P_{N N D}(p)$ and $P_{N R D}(p)$ do not depend on $S$ and $A$. Hence in the remaining part of the proof we take $S=A=1$.

Let us first consider the NND model. By symmetry, let us assume without loss of generality that a given transmitter (located at the origin) sends a packet to its nearest neighbor to the right on the real line without knowing if this node is silent or not. By the known property of the Poisson point process, the distance $R$ to this node has an exponential distribution with parameter $\lambda$. Moreover given $R=r$, all other nodes form a Poisson point process of intensity $\lambda$ on $(-\infty, 0) \cup(r, \infty)$. Conditioning on $R=r$ the probability of the SINR condition (2.2) is equal to:

$$
\begin{aligned}
\mathbf{P}\left\{F S \geq T I_{\Phi^{1}} l(r) \mid R=r\right\} & =\int_{0}^{\infty} e^{-s T l(r)} \mathrm{d} \mathbf{P}\left(I_{\Phi} \leq s \mid R=r\right) \\
& =\psi_{I_{\Phi^{1}} \mid R=r}(T l(r)),
\end{aligned}
$$

where $\psi_{I_{\Phi^{1}} \mid R=r}$ denotes the Laplace transform of the shot noise generated at the location of the receiver by a Poisson point process of intensity $p \lambda$ on $(-\infty, 0) \cup(r, \infty)$. It is know (it can be derived from the formula for the Laplace functional of the Poisson p.p. (see e.g. [3])):

$$
\psi_{I_{\Phi^{1}} \mid R=r}(\xi)=\exp \left\{-\lambda p\left(\int_{r}^{\infty}+\int_{0}^{\infty}\right)\left(1-\mathbf{E}\left[e^{-\xi F / l(s)}\right]\right) \mathrm{d} s\right\} .
$$

Plugging in the conditional probability of the SINR condition and unconditioning with respect to $R$ we obtain:

$$
\begin{aligned}
P_{N N D}(p)=\lambda(1-p) \int_{0}^{\infty} & e^{-\lambda r} \exp \left\{-\lambda p\left(\int_{r}^{\infty}+\int_{0}^{\infty}\right)\right. \\
& \left.\left(1-\mathbf{E}\left[e^{-l(r) T F / l(s)}\right]\right) \mathrm{d} s\right\} \mathrm{d} r
\end{aligned}
$$

where $(1-p)$ in front of the first integral is due to the fact that successful reception is possible only if the nearest node is not authorized to transmit at the given time slot. Assuming exponential fading $F$ and the power law path-loss $l(\cdot)$ we evaluate $1-\mathbf{E}\left[e^{-l(r) T F / l(s)}\right]=\frac{1}{1+(s / r)^{\beta} / T}$ and obtain

$P_{N N D}(p)=\lambda(1-p) \int_{0}^{\infty} e^{-\lambda r\left(1+p T^{1 / \beta}\left(C\left(T^{-1 / \beta}, \beta\right)+C(\beta)\right)\right)} \mathrm{d} r$,

which boils down to the right hand side of (2.4). In order to obtain expression (2.5) for the $N R D$ model we follow the same arguments, with the following modifications. The distance to the nearest receiver to the right has the exponential distribution of parameter $\lambda(1-p)$. Moreover the distribution of the point process of emitters is Poisson with parameter $\lambda p$ and independent of the location of this receiver. Note also that by the very choice of the receiver, it is not authorized to emit during the given time slot, hence there is no $(1-p)$ factor in the numerator.
Remark 2.2: It is easy to see that $\mathcal{C}_{2}-1 \leq \mathcal{C}_{1}$ and hence $P_{N R D} \geq P_{N N D}$, i.e., the opportunistic choice of the receiver pays off regarding the likelihood of successful transmission.

\section{F. Density of progress}

Now we will calculate and optimize in $p$ the mean density of progress $d$ defined as the expected total progress of all the successful transmissions per unit of road length;

$d=\mathbf{E}\left[\sum_{X_{i} \in[0,1]}\left|X_{i}-Y_{i}\right| \mathbb{I}\left(e_{X_{i}}=1\right) \mathbb{I}\left(e_{Y_{i}}=0\right) \mathbb{I}\left(\operatorname{SINR}_{\left(X_{i}, Y_{i}\right)} \geq T\right)\right]$,

where $Y_{i}$ is the receiver chosen by the transmitter $X_{i}$ in the given model (NND or NRD) ${ }^{1}$. The density of progress, can also be seen as quantifying the number of bit-(or packet)-meters "pumped" per unit length of the VANET network. Thus we see it as a generic performance metric of value-added services generating point-to-point traffic.

Proposition 2.3: Under the assumptions of Proposition 2.1 the density of progress in the NND receiver model is equal to:

$$
d=d_{N N D}(p)=\frac{p(1-p)}{\left(1+p \mathcal{C}_{1}\right)^{2}}
$$

and is maximized for $p$ equal to:

$$
p_{N N D}^{*}=\frac{\mathcal{C}_{1}+1-\sqrt{\mathcal{C}_{1}^{2}-1}}{2 \mathcal{C}_{1}} .
$$

Moreover, $0<p_{N N D}^{*}<1$.

Proof: Using Campbell's formula, the density of progress in the NDD model can be expressed as:

$$
d_{N N D}(p)=\lambda p(1-p) \mathbf{E}\left[R \mathbf{P}\left\{\mathbb{I}\left(F S \geq T I_{\Phi^{1}} l(r) \mid R=r\right\}\right]\right.
$$

with the notation as in the proof of Proposition 2.1. Following the same arguments as in this latter proof we obtain

$$
d_{N N D}(p)=\lambda^{2} p(1-p) \int_{0}^{\infty} r e^{-\lambda r\left(1+p \mathcal{C}_{1}\right)} \mathrm{d} r
$$

which is equal to the right-hand side of (2.6). Taking the derivative of the latter expression in $p$ we find that its sign is equal to that of the polynomial $P(p)=1-p\left(2+\mathcal{C}_{1}\right)-2 p^{2} \mathcal{C}_{1}^{2}+2 p^{3} \mathcal{C}_{1}^{2}$. Note that $P(-\infty)=-\infty, P(0)=1, P(1)=-1-\mathcal{C}_{1}<0$ and $P(\infty)=\infty$. Hence in the interval $(0,1), P(\cdot)$ has a unique root: $p_{N N D}^{*}$ which maximizes $d_{N N D}(p)$. The explicit expression for this root follows from the general formulas for the roots of cubic equations.

Proposition 2.4: Under the assumptions of Proposition 2.1 the density of progress in the NRD receiver model is equal to:

$$
d_{N R D}(p)=\frac{p(1-p)}{\left(1+p\left(\mathcal{C}_{2}-1\right)\right)^{2}} .
$$

and is maximized for $p$ equal to:

$$
p_{N R D}^{*}=\frac{\mathcal{C}_{2}+1-\sqrt{\mathcal{C}_{2}^{2}-1}}{2 \mathcal{C}_{2}} .
$$

Moreover, $0<p_{N R D}^{*}<1$.

${ }^{1} \mathbb{I}($ Condition $A)$ is the indicator function. The value of this function is 1 if Condition $A$ is satisfied and 0 otherwise. 
Proof: Using the same reasoning as in the proof of Proposition 2.3 we find expression (2.7) and its optimization in $p$ goes along the same lines as for the NND model replacing $C_{1}$ by $C_{2}$.

\section{G. Nearest neighbor warning delay time}

In this section we are interested in emergency broadcasting in VANETs. Suppose that a typical (tagged) node needs to warn its nearest neighbor in a given direction by sending it a specific packet. Then $P_{N N D}(p)$ given by (2.4) is the probability that this task can be accomplished with a single transmission. However, a single transmission might not be sufficient. Hence, we assume that the tagged node does not have to respect the Aloha scheme and after the first unsuccessful transmission it is allowed to retransmit the packet in successive slots, possibly several times, until it has been received successfully. Regarding the geographic displacement of nodes during this process, we assume that it is small enough to only impact on the fading conditions and not the average path-loss conditions. In other words, we assume Poisson locations of nodes $X_{i} \in \Phi$ unchanged and the fading $F_{(x, y)}^{n}$, independently re-sampled for every consecutive retransmission slot $n=1,2, \ldots$, for every transmitter-receiver pair $(x, y)$.

Let us denote by $L_{0}$ the number of consecutive time slots needed for the tagged node to successfully transmit the emergency message to its nearest neighbor in $\Phi$ in a given direction. We call $L_{0}$ emergency delay at the tagged node. Since we have assumed that fading is independent across the time slots, given $\Phi, L_{0}$ is a geometric random variable with mean $1 / \pi(\Phi)$, where $\pi(\Phi)$ is the probability of successful reception in a given time slot, given the location of nodes $\Phi$. (This latter conditional probability regards only uncertainty due to fading conditions and MAC statuses of nodes in the network.)

In what follows we will give the expression for the unconditional expected emergency delay $\mathbf{E}\left[L_{0}(\Phi)\right]$, i.e.; average emergency delay at the tagged node, where the averaging regards all possible Poisson configurations of other nodes $\Phi$. As we will see, this expectation is finite only if $p$ is sufficiently small for any given $T$ and $\beta$.

Let us denote

$$
\begin{aligned}
\mathcal{D}_{1}(p)=\mathcal{D}_{1}(p ; T, \beta)=T^{1 / \beta} & \left(\int_{T^{-1 / \beta}}^{\infty} \frac{1}{u^{\beta}+1-p} \mathrm{~d} u\right. \\
& \left.+\int_{0}^{\infty} \frac{1}{u^{\beta}+1-p} \mathrm{~d} u\right)
\end{aligned}
$$

and

$$
\mathcal{D}_{2}(p)=\mathcal{D}_{2}(p ; T, \beta)=2 T^{1 / \beta} \int_{0}^{\infty} \frac{1}{u^{\beta}+1-p} \mathrm{~d} u .
$$

Proposition 2.5: Under the assumptions of Proposition 2.1, the average emergency delay in the MMD model is equal to

$$
\mathbf{E}\left[L_{0}\right]=\frac{1}{(1-p)\left(1-p \mathcal{D}_{1}(p)\right)}
$$

provided

$$
p \mathcal{D}_{1}(p)<1
$$

and $\mathbf{E}\left[L_{0}\right]=\infty$ otherwise.
Proof: We need to evaluate $\pi(\Phi)$, i.e. the probability of successful reception in a given time slot, given $\Phi$. As previously in the NND model, we suppose that the receiver (nearest neigbour) is at distance $r$ from the tagged node. Following the same arguments as in the proof of Proposition 2.1, we find that $\pi(\Phi)$ given the receiver at distance $r$, which we denote by $\pi(r, \Phi)$ is equal to $(1-p) \mathbf{E}\left[e^{-l(r) T I(r, \Phi)} \mid \Phi\right]$, where $I(r, \Phi)$ is the interference at the receiver. Hence $\pi(\Phi)$ can be expressed as a conditional Laplace transform of the shot-noise given $\Phi$. An explicit expression for this is known (cf. the proof of [4, Lemma 17.30]) and in our case gives

$$
\pi(r, \Phi)=(1-p) \exp \left\{\sum_{X_{i} \in \Phi} \log \left(1-\frac{p}{1 / T\left(\left|X_{i}\right| / r\right)^{\beta}+1}\right)\right\} .
$$

Taking the expectation of the inverse of the latter expression with respect to the distribution of the homogeneous Poisson process of intensity $\lambda p$ truncated to $(-\infty, 0) \cup(r, \infty)$, again by the known expression for the Laplace transform of a shotnoise, we obtain

$$
\begin{aligned}
& \mathbf{E}\left[\frac{1}{\pi(r, \Phi)} \mid R=r\right]=\frac{1}{1-p} \exp \left\{-\lambda\left(\int_{r}^{\infty}+\int_{0}^{\infty}\right)\right. \\
& \left.\left(1-\frac{1}{1-\frac{p}{1 / T(s / r)^{\beta}+1}}\right) \mathrm{d} s\right\}=\frac{\exp \left\{\lambda \operatorname{pr} \mathcal{D}_{1}(p)\right\}}{1-p} .
\end{aligned}
$$

Consequently

$$
\begin{aligned}
\mathbf{E}\left[L_{0}\right] & =\frac{\lambda}{1-p} \int_{0}^{\infty} e^{-\lambda r} e^{\lambda p r \mathcal{D}_{1}(p)} \mathrm{d} r \\
& =\frac{\lambda}{1-p} \int_{0}^{\infty} e^{-\lambda r\left(1-p \mathcal{D}_{1}(p)\right)} \mathrm{d} r
\end{aligned}
$$

It is easy to see that this integral is infinite if $p \mathcal{D}_{1}(p) \geq 1$ and $1 /\left((1-p)\left(1-p \mathcal{D}_{1}(p)\right)\right)$ otherwise. This completes the proof.

Remark 2.6: Note that $\mathcal{D}_{1}(p) \geq \mathcal{C}_{1}$ with the equality reached when $p \rightarrow 0$. Hence, for small $p$ we have $\mathcal{D}_{1}(p) \approx \mathcal{C}_{1}$, and the critical $p$ for which the average expected delay explodes is approximately $1 / \mathcal{C}_{1}$.

\section{H. Neighborhood discovery time}

Let us now consider another important type of communication in VANETs, related to position broadcasting. Assume that each node uses a fraction $p^{\prime}$ of the time slots in which it is authorized by Aloha to transmit, to broadcast a special packet to inform every other node being able to receive it about the transmitter's position (and perhaps other nodes' parameters). More precisely, for each transmission slot, a given node transmits such a special packet (called the localisation packet) independently, with probability $p^{\prime}$ and other (usual traffic) packets sent with probability $1-p^{\prime}$. We are interested in the mean time required for a given (typical) node to discover all nodes within a given distance $R$ from it by receiving at least one localisation packet from each of them.

We denote by $D_{i}$ the time (number of time slots) until the reception of the first localisation packet from node $X_{i}$ by the typical node located at the origin. There is no explicit expression for (the mean of) the maximum $\max _{X_{i} \in[-R, R]} D_{i}$ 
however, we can give an explicit expression for the expectation of the sum $L_{d i s c}(R):=\sum_{X_{i} \in[-R, R]} D_{i}$, which is in upper bounded by the aforementioned maximum.

Proposition 2.7: Assume exponential fading and negligible noise $(W=0)$. Then for any $R>0$

$$
\mathbf{E}\left[L_{d i c s}(R)\right]=\frac{2}{p^{\prime}(1-p) p^{2} \mathcal{D}_{2}(p)}\left(e^{\lambda p R D_{2}(p)}-1\right),
$$

where $D_{2}(p)=D_{2}(p ; T, \beta)$ is as defined in Section II-G.

Proof: By Campbell's formula

$$
\mathbf{E}\left[L_{d i s c}(R)\right]=\lambda \int_{-R}^{R} \mathbf{E}\left[D_{r}\right] \mathrm{d} r=\lambda \int_{-R}^{R} \mathbf{E}\left[\frac{1}{p^{\prime} p \pi(r, \Phi)}\right] \mathrm{d} r,
$$

where $\pi(r, \Phi)$ is given in the proof of Proposition 2.5. Consequently

$$
\mathbf{E}\left[L_{d i s c}(R)\right]=\frac{\lambda}{p p^{\prime}(1-p)} \int_{-R}^{R} e^{\lambda p r D_{2}(p)} \mathrm{d} r .
$$

Evaluation of the above integral concludes the proof.

\section{IMPACT OF EXTERNAL NOISE}

In the previous section we assumed that the external noise was negligible $(W=0)$. In this section we study the impact of a non-null external noise $W$. It is easy to extend the results of Propositions 2.1, 2.3 and 2.4 to the case with non-null noise. The optimisation of the density of progress is however not explicit.

Proposition 3.1: Under the assumptions of Proposition 2.1 for the NND receiver model, with an arbitrary noise distribution $W$, we have the followin probability of successful reception:

$$
p_{N N D}=\lambda(1-p) \int_{0}^{\infty} e^{-r \lambda\left(1+p \mathcal{C}_{1}\right)-T W(A r)^{\beta}} \mathrm{d} r
$$

and the density of progress:

$$
d_{N N D}=\lambda(1-p) \int_{0}^{\infty} r e^{-r \lambda\left(1+p \mathcal{C}_{1}\right)-T W(A r)^{\beta}} \mathrm{d} r .
$$

Replacing $\mathcal{C}_{1}$ by $\mathcal{C}_{1}-1$ in the two above expressions we obtain, respectively, the probability of successful transmission $p_{N R D}$ and the density of progress $d_{N R D}$ in the NRD receiver model with an arbitrary noise $W$.

Proof: The proof follows the same lines as in the proofs of Propositions 2.1, 2.3 and 2.4.

The introduction of noise into the computation of the delay and the neighborhood discovery time is similar and is not carried out in this paper for reasons of space.

\section{NUMERICAL EXAMPLES}

The default parameters are $\lambda=0.01$ vehicles per meter (the mean inter-vehicle distance is equal to 100 meters), $\beta=4$, $T=1$ and $A=1$. The fading is exponentially distributed with rate 1 .

In Figure 1 we show the capture probabilities for $P_{N N D}$ and $P_{N R D}$. We observe that the results obtained are close; for the same transmission probability $p, P_{N N D}$ is sligthly greater than $P_{N R D}$. We also notice that these probabilities are greater for $\beta=4$ than for $\beta=2$. This is because when $\beta$ is larger, then interference decays more rapidly.

In Figure 2 we present the densities of progress $d_{N N D}$ and $d_{N R D}$. We note that for the same transmission probability $p$ we have $d_{N R D}>d_{N N D}$ which confirms the inequality we show in general. For $\beta=2$ the maximum of $d$ is around $2.5 \%$ greater for the NRD scheme than for the NND scheme. For $\beta=4$ the maximum of $d$ is around $5 \%$ greater for the NRD scheme than for the NND scheme. Thus we can conclude that the opportunism of the NRD scheme has a limited impact.

In Figure 3 we present the value of $p$ which optimizes the density of progress for both the NND and NRD schemes. We observe that there is a significant difference for this value of $p$ for the two schemes and this difference increases with $\beta$.

In Figure 4 we present the value of the average delay with respect to $p$ for $\beta=2,3,4$ and 5 and $T=10$. We note that there is a critical value of $p$ ( which depends on $\beta$ ) for which this delay becomes infinite. Logically this critical value of $p$ is an increasing function of $\beta$. With less interference the transmission probability $p$ can be higher. Figure 5 also gives the value of the average delay with respect to $p$ for $T=1,2,5,10$. Of course for larger $T$ the critical value of $p$ is smaller.

In Figure 6 we present the density of progress $d_{N N D}$ with respect to $p$ for $W=$ $10^{-11}, 10^{-10}, 10^{-9}, 10^{-8}, 10^{-7}, 10^{-6}, 10^{-5}$. We note that the noise has a very great impact on the network performances. For $W \geq 10^{-7}$ the density of progress is more than 10 times smaller than without noise.

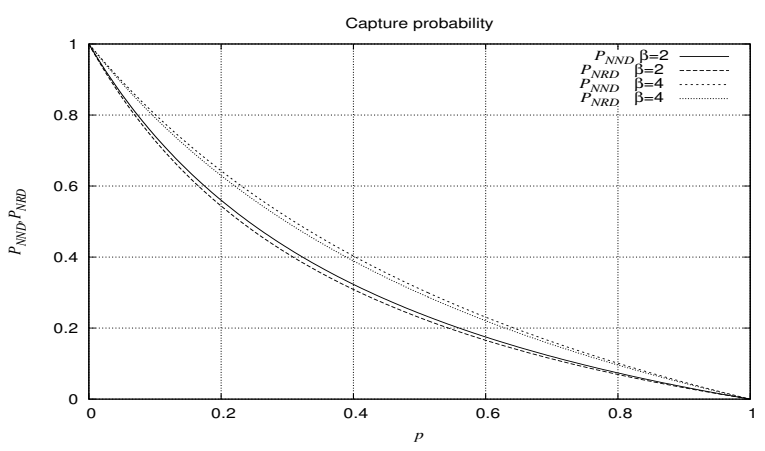

Fig. 1. Capture probabilities $P_{N N D}$ and $P_{N R D}$ for $\beta=2$ and $\beta=4$

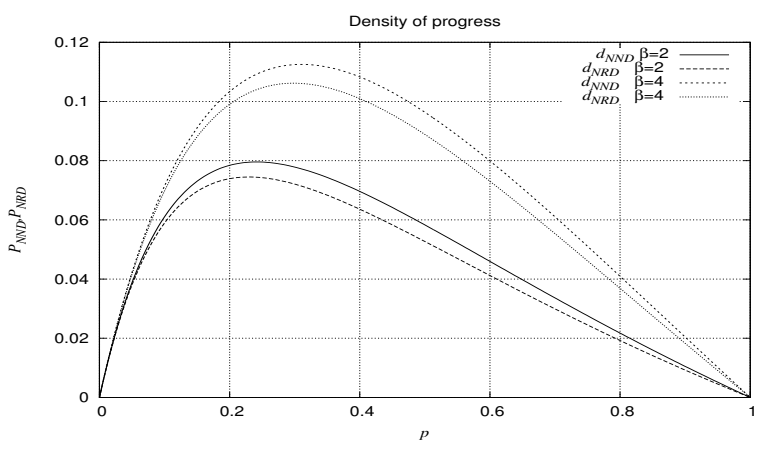

Fig. 2. Density of successful transmissions for $\beta=2$ and $\beta=4$ 


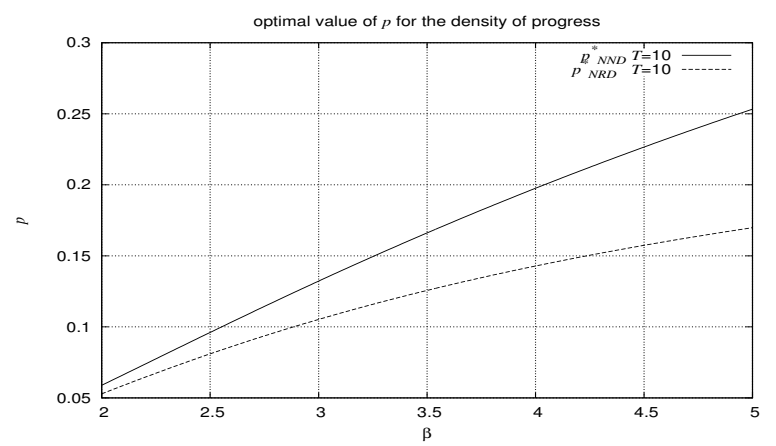

Fig. 3. Optimal value of $p$ for the density of successful transmissions for $2 \leq \beta \leq 5$

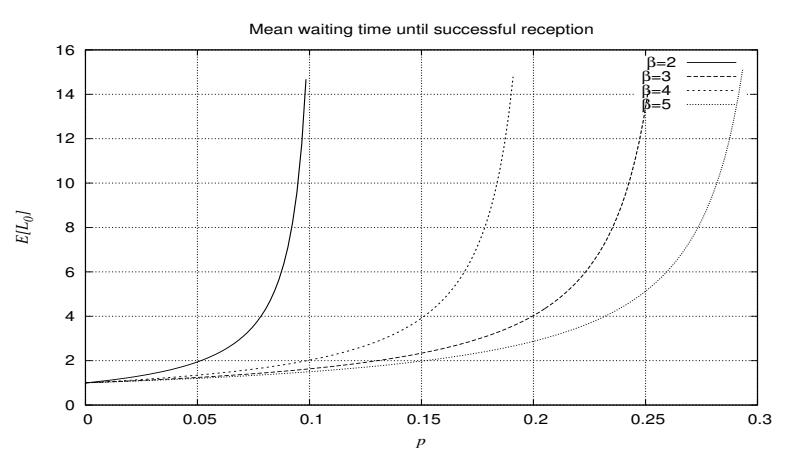

Fig. 4. Expected delay until successful reception $\mathbf{E}\left[L_{0}\right]$ for $\beta=2,3,4,5$.

\section{CONCLUSION}

In this paper we have studied the performance of two simple NND and NRD relaying schemes in linear networks. We have derived closed formulas for the capture probability, the density of progress and the average delay. These formulas allow the influence of the models parameters to be easily studied. We show how to introduce noise in our evaluation and we obtain other formulas that can be numerically evaluated.

\section{REFERENCES}

[1] Vinel A, Vishnevsky V, and Koucheryavy Y. A simple analytical model for the periodic broadcasting in vehicular ad-hoc networks. GLOBECOM Workshops, pages 1-5, 30 Dec 2008.

[2] Emmanuel Baccelli, Philippe Jacquet, Bernard Mans, and Georgios Rodolakis. Highway Vehicular Delay Tolerant Networks: Information Propagation Speed Properties. IEEE Transactions on Information Theory. Vol 58, No 3 pages 1743 - 1756, March 2012.

[3] F. Baccelli and B. Błaszczyszyn. Stochastic Geometry and Wireless Networks, Volume I - Theory, volume 3, No 3-4 of Foundations and Trends in Networking. NoW Publishers, 2009.

[4] F. Baccelli and B. Błaszczyszyn. Stochastic Geometry and Wireless Networks, Volume II - Applications, volume 4, No 1-2 of Foundations and Trends in Networking. NoW Publishers, 2009.

[5] F. Baccelli, B. Blaszczyszyn, and P. Mühlethaler. An Aloha Protocol for Multihop Mobile Wireless Networks.

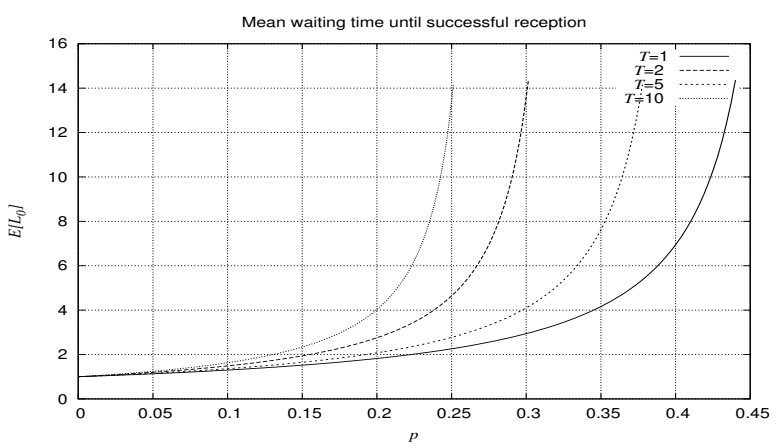

Fig. 5. Expected delay until successful reception $\mathbf{E}\left[L_{0}\right]$ for $T=1,2,5,10$.

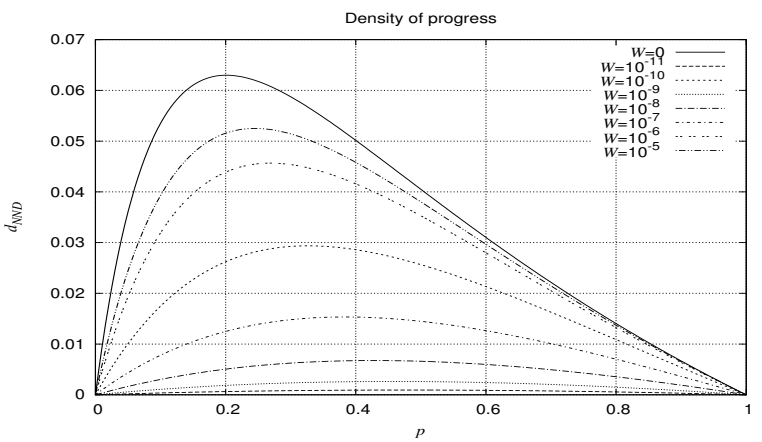

Fig. 6. Expected delay until successful reception $\mathbf{E}\left[L_{0}\right]$ for various values of the noise.

In Proceedings of the Allerton Conference, University of Illinois, Urbana Champaign, November 2003. And in IEEE Transactions on Information Theory, Vol 52, No 2: pages 421-436, February 2006.

[6] F. Baccelli, B. Błaszczyszyn, and P. Mühlethaler. Stochastic analysis of spatial and opportunistic Aloha. IEEE JSAC, special issue on Stochastic Geometry and Random Graphs for Wireless Networks, pages 1105-1119, 2009.

[7] B. Blaszczyszyn, P. Muhlethaler, and Y. Toor. Maximizing Throughput of Linear Vehicular Ad-hoc NETworks (VANETs) - a Stochastic Approach. In Proc. of European Wireless 2009, May 2009. Aalborg, Denmark.

[8] H.A. Cozzetti, R.M. Scopigno, L. Casone, and G. Barba. Comparative analysis of IEEE 802.11p and MS-Aloha in VANET scenarios. In Proc. of Services Computing Conference (APSCC), pages 64-69, Singapore, Dec 2009.

[9] M. Haenggi. Outage, local throughput, and capacity of random wireless networks. IEEE Trans. Wireless Comm., Vol 8: pages 4350-4359, August 2009.

[10] R. Reinders, E. M. van Eenennaam, G. Karagiannis, and G. J. Heijenk. Contention window analysis for beaconing in vanets. In Seventh IEEE International Wireless Communications and Mobile Computing conference, IWCMC 2011, Istanbul, Turkey, pages 1481-1487, Istanbul, July 2011. IEEE Computer Society.

[11] Razvan Stanica, Emmanuel Chaput, and Andr-Luc Beylot. Why VANET Beaconing is More than Simple Broadcast. In IEEE Vehicular Technology Conference (VTC), San Francisco, pages 1-5, September 2011. 